\title{
A Case of Splenic Abscess Complicated by Infective Endocarditis
}

\author{
Nobuhiro Takeuchi, ${ }^{1}$ Kazumasa Emori, ${ }^{1}$ Makoto Yoshitani, Junichi Soneda, \\ Atsuyoshi lida, ${ }^{2}$ Hiromichi Naito, ${ }^{2}$ Tetsuya Yumoto, ${ }^{2}$ Kohei Tsukahara, ${ }^{2}$ and Atsunori Nakao ${ }^{2}$
}

\begin{abstract}
Background: Splenic abscess is a rare but well-documented complication of infective endocarditis. We report a case of splenic abscess associated with infective endocarditis with evidence of sepsis, vegetation, and positive blood cultures.

Case Presentation: A 92-year-old female presented to our department with a high-grade fever and disturbance of consciousness. Laboratory data revealed leukocytosis and an elevation of C-reactive protein. Computed tomography and ultrasonography revealed a cystic mass at the lower lobe of the spleen that measured $5 \mathrm{~cm}$ in diameter. On the basis of images and laboratory data of leukocytosis, a diagnosis of septic shock associated with splenic abscess was made. Antibiotics and splenectomy alleviated the patient's inflammatory reactions and improved her septic shock. Blood culture at arrival was positive for Escherichia coli. Transesophageal echocardiography was performed, revealing an $8-\mathrm{mm}$ vegetation at the anterior leaflet of the mitral valve without any evidence of congestive heart failure. Administration of cefmetazole $2 \mathrm{~g} / \mathrm{d}$ was continued for 28 days until the patient's inflammatory reactions improved and the vegetation disappeared.
\end{abstract}

Conclusion: Physicians should always be aware of potential infective endocarditis whenever splenic abscess is encountered.

Keywords: Escherichia coli; infective endocarditis; splenic abscess

$\mathbf{S}$ PLENIC ABSCESS is a relatively rare but well-documented condition. With the recent development of imaging modalities and the increasing number of immunosuppressive hosts that can be treated with chemotherapy or hemodialysis, the number of patients with splenic abscess is increasing. Signs of splenic abscess can be hidden and diagnosis can be considerably difficult, even for experienced clinicians. Rapid diagnosis and treatment are imperative, because the condition can become fatal. Splenic abscess develops because of bacteremic seeding of an infarcted splenic zone secondary to embolized vegetations, or more directly through seeding of the spleen by infected embolized heart valve vegetations [1].

Splenic abscess can be a rare complication of infective endocarditis [2]. Splenic abscess accompanied by infective endocarditis can cause sepsis and become fatal if left untreated. Herein, we report a case of splenic abscess accom- panied by infective endocarditis cured by splenectomy [3]. Sharing our experience may help emergency physicians make appropriate diagnostic/therapeutic strategies.

\section{Case Presentation}

A 92-year-old female presented to our hospital with symptoms of a high-grade fever and disturbance of consciousness. She had been well until the onset of these symptoms. She had no history of diabetes mellitus, malignant tumors, or collagen disease. The patient denied any use of tobacco or alcohol. Upon arrival, her blood pressure was 83/ $49 \mathrm{~mm} \mathrm{Hg}$, oxygen saturation was $98 \%$ under $5 \mathrm{~L} / \mathrm{min}$ oxygen, and her body temperature was $39.0^{\circ} \mathrm{C}$. She was believed to have septic shock. Her abdomen was distended with normal peristalsis. Mild tenderness was found on the left upper

\footnotetext{
${ }^{1}$ Department of Internal Medicine, Kobe Tokushukai Hospital, Tarumi, Kobe, Japan.

${ }^{2}$ Department of Critical Care and Emergency Medicine, Okayama University Hospital, Okayama, Japan.

(C) Nobuhiro Takeuchi et al. 2016; Published by Mary Ann Liebert, Inc. This Open Access article is distributed under the terms of the Creative Commons License (http://creativecommons.org/licenses/by/4.0), which permits unrestricted use, distribution, and reproduction in any medium, provided the original work is properly credited.
} 
abdomen. No mass was palpable and no sign of peritoneal irritation was observed over the abdomen. Blood tests revealed leukocytosis (white blood cell count of 70,470 per microliter with $96.0 \%$ neutrophils). Blood chemistry analyses demonstrated elevated C-reactive protein $(22.99 \mathrm{mg} / \mathrm{dL})$, moderately decreased total protein $(6.0 \mathrm{~g} / \mathrm{dL})$, moderately decreased albumin $(2.0 \mathrm{~g} / \mathrm{dL})$, mildly increased lactate dehydrogenase (272 IU/L), and mildly increased creatine kinase (265 IU/L); coagulant dysfunction (60\% prothrombin), and impaired glucose tolerance $(121 \mathrm{mg} / \mathrm{dL})$. Furthermore, moderately impaired kidney function $(2.58 \mathrm{mg} / \mathrm{dL}$ serum creatine; blood urea nitrogen $26.7 \mathrm{mg} / \mathrm{dL})$, and hypokalemia $(2.9 \mathrm{mEq} / \mathrm{mL})$ were also found.

Abdominal radiography revealed normal gas distribution. Contrast computed tomography (CT) revealed a $59 \times 65 \mathrm{~mm}$ low-density mass at the lower spleen (Fig. 1A). Ultrasonography revealed a cloudy low echoic lesion of the lower spleen. Because a clinical diagnosis of splenic abscess was made, cefmetazole $2 \mathrm{~g} / \mathrm{d}$ was administered immediately after admission. Two sets of blood cultures at arrival were positive for Escherichia coli and sensitive to cefmetazole. To rule out infective endocarditis, transthoracic echocardiography was performed and failed to detect vegetation. However, transesophageal echocardiography demonstrated an 8-mm vegetation at the anterior leaflet of the mitral valve without any evidence of congestive heart failure (Fig. 1B). On day three after admission, splenectomy was performed under general anesthesia to alleviate her inflammatory reactions and improve septic shock. No cardiac surgery was performed because of the small-sized vegetation without symptoms. Resected specimen revealed a 5-cm cystic lesion at the lower lobe of the spleen. A microscopic specimen revealed necrosis and neutrophil cells, a finding consistent with splenic abscess. The patient's post-operative course was uneventful. Administration of cefmetazole $2 \mathrm{~g} / \mathrm{d}$ was continued for $28 \mathrm{~d}$; thereafter, chemical analyses of inflammatory response improved dramatically and the patient was discharged.

\section{Discussion}

Splenic abscess is associated with high morbidity and mortality. Splenic tissue is extremely delicate, especially when

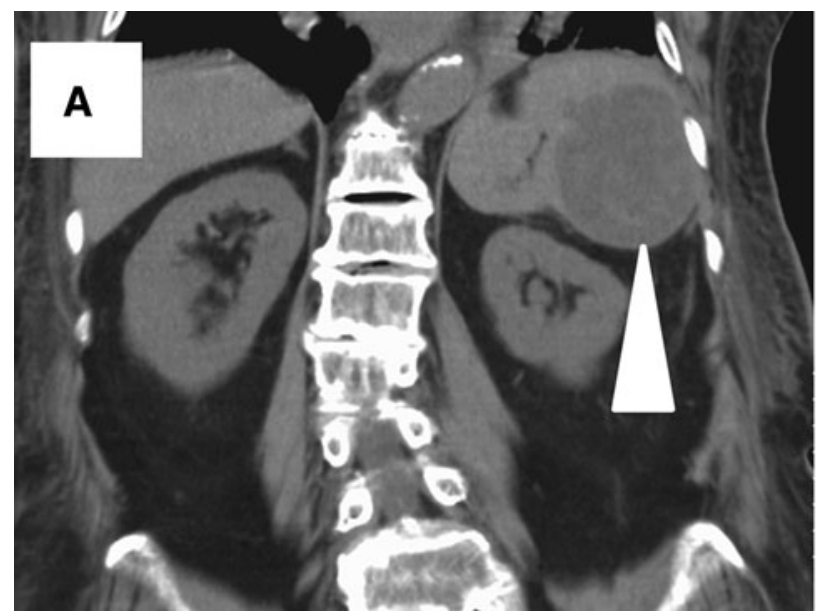

the abscess is in a sub-capsular location, and minimal trauma can cause splenic rupture. Therefore, early identification of symptoms and swift treatment are essential for a favorable outcome. Splenic abscess accompanied by infective endocarditis is reportedly relatively rare, accounting for $4.8 \%$ of patients with splenic abscess [4]. Septic emboli, generally observed in patients with left-sided infective endocarditis, to the spleen may contribute to the pathogenesis of splenic infarction. Splenic abscess or infarction may be encountered at different stages in patients with infective endocarditis caused by septic emboli of the spleen. Splenic abscess will eventually develop in approximately $5 \%$ of patients with splenic infarction [4]. Embolic phenomena are not restricted to the spleen and are sometimes extended to the brain.

Clinical presentation of splenic abscess includes highgrade fever, left-sided upper abdominal pain, and splenomegaly. However, many cases of splenic abscess present with nonspecific complaints. Therefore, imaging modalities, including ultrasonography or $\mathrm{CT}$, are crucial for diagnosing splenic abscess. If splenic abscess is left untreated, the abscess may possibly rupture, leading to fatal conditions, including hemorrhagic shock or peritonitis. Whenever clinicians encounter a high-grade fever or infectious disease with unknown origins, they should investigate infectious origins with imaging modalities, including abdominal CT scan or magnetic resonance imaging, and suspect splenic abscess as one of the differential diagnoses. Causative agents of splenic abscess include gram-positive cocci (notably, Staphylococcus aureus and Streptococcus pyogenes) and gram-negative bacilli (Klebsiella pneumoniae, Escherichia coli, Pseudomonas species, Salmonella species, and Proteus). Staphylococcus aureus is the primary organism in $50 \%$ of cases with splenic embolization/abscess.

There is no consensus about the operative timing of splenic abscess resection, and valve replacement in patients with splenic abscess is complicated by infectious endocarditis. If valve replacement is prioritized, there is a possibility that prosthetic valve infection might occur because of the presence of splenic abscess. In addition, the risk of intra-abdominal hemorrhage from anti-coagulation during cardiopulmonary bypass and the potential seeding of the prosthetic valve with micro-organisms originating from a splenic abscess may exist.

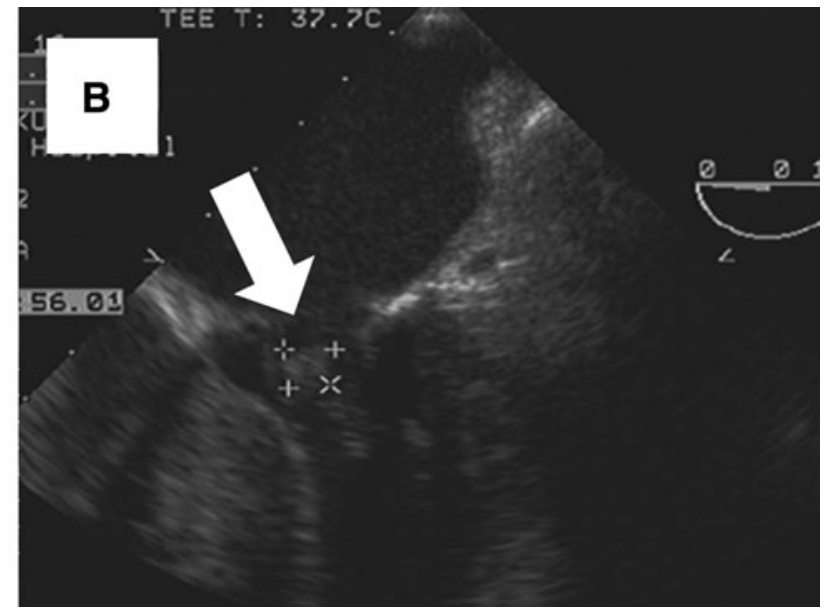

FIG. 1. (A) Computed tomography revealed a $59 \times 65 \mathrm{~mm}$ low-density mass at the lower spleen (black arrow). (B) Transesophageal echocardiography revealed an $8-\mathrm{mm}$ vegetation at the anterior leaflet (white arrowhead). 
On the other hand, if splenectomy is prioritized, patients might become immunocompromised or develop a tendency to bleed. If the patient's general state allows it, performing splenectomy prior to valve replacement surgery is ideal to prevent re-infection of the valve prosthesis [5]. Successful one-stage splenectomy procedures combined with valve replacement have also been reported [6]. According to guidelines for surgical candidates with native valve endocarditis [7], surgical treatment is indicated for patients with valve stenosis or regurgitation resulting in heart failure, recurrent emboli, and persistent vegetations despite appropriate antibiotic treatment, or mobile vegetations greater than $10 \mathrm{~mm}$ with or without emboli. In our patient, the size of the vegetation was less than $10 \mathrm{~mm}$ and there was no evidence of worsening heart failure, therefore, conservative treatment with antibacterial drugs was considered optimal.

The number of reports focusing on the significance of splenic abscess drainage is increasing. Percutaneous drainage under ultrasonography or CT guidance is an option for highrisk patients and those for whom major surgery is contraindicated. Chou et al. [8] reported the efficiency of splenic abscess drainage with a lesion of more than $3.5 \mathrm{~cm}$ or the placement of multiple drainage tubes in multiple abscesses. In some cases, treatment of recurrence of splenic abscess may be possible simply by abscess drainage. In cases in which abscess drainage fails, surgical removal of the spleen is crucial. Ting et al. [1] reported that the indications for splenic resection of splenic abscess are (1) refractory septicemia, (2) abscess with high risk of rupture, (3) abscess that is located in margins of spleen, or (4) abscess larger than $2 \mathrm{~cm}$.

\section{Conclusion}

We report a case of splenic abscess complicated by infective endocarditis. For every diagnosis of splenic abscess, scrutiny regarding the possible source of emboli should be exercised. On the other hand, a high index of clinical suspicion of splenic abscess should be maintained while treating patients with infective endocarditis.

\section{Author Disclosure Statement}

No competing financial interests exist.

\section{References}

1. Ting W, Silverman NA, Arzouman DA, et al. Splenic septic emboli in endocarditis. Circulation 1990;82:IV105-109.
2. Elasfar A, AlBaradai A, AlHarfi Z, et al. Splenic abscess associated with infective endocarditis: Case series. J Saudi Heart Assoc 2015;27:210-215.

3. Wang CC, Lee $\mathrm{CH}$, Chan CY, et al. Splenic infarction and abscess complicating infective endocarditis. Am J Emerg Med 2009;27:1021.e1023-1025

4. Robinson SL, Saxe JM, Lucas CE, et al. Splenic abscess associated with endocarditis. Surgery 1992;112:781-786.

5. Ebels J, Van Elst F, Vanderveken M, et al. Splenic abscess complicating infective endocarditis: Three case reports. Acta Chir Belg 2007;107:720-723.

6. Yoshikai M, Kamachi M, Kobayashi K, et al. Splenic abscess associated with active infective endocarditis. Jpn J Thorac Cardiovasc Surg 2002;50:478-480.

7. Horstkotte D, Follath F, Gutschik E, et al. Guidelines on prevention, diagnosis and treatment of infective endocarditis executive summary: The task force on infective endocarditis of the European Society of Cardiology. Eur Heart J 2004;25: 267-276.

8. Chou YH, Tiu CM, Chiou HJ, et al. Ultrasound-guided interventional procedures in splenic abscesses. Eur J Radiol 1998;28:167-170.

Address correspondence to: Dr. Atsunori Nakao

Department of Emergency and Critical Care Medicine Okayama University Graduate School of Medicine Dentistry and Pharmaceutical Sciences 2-5-1 Shikata-cho Kita-ku, Okayama-shi Okayama 700-8558 Japan

E-mail: qq-nakao@okayama-u.ac.jp
Abbreviation Used
$\mathrm{CT}=$ computed tomography

Cite this article as: Takeuchi N, Emori K, Yoshitani M, Soneda J, Iida A, Naito H, Yumoto T, Tsukahara K, Nakao A (2016) A case of splenic abscess complicated by infective endocarditis. Surgical Infections Case Reports 1:1, 139-141, DOI: 10.1089/crsi.2016.0036 